\title{
Photon-number fluctuation and correlation of bound soliton pairs in mode-locked fiber lasers
}

\author{
Ray-Kuang Lee \\ Department of Photonics and Institute of Electro-Optical Engineering, National Chiao-Tung University, \\ Hsinchu 300, Taiwan, and Department of Electrical Engineering and Institute of Photonics Technologies, \\ National Tsing-Hua University, Hsinchu 300, Taiwan \\ Yinchieh Lai \\ Department of Photonics and Institute of Electro-Optical Engineering, National Chiao-Tung University, \\ Hsinchu 300, Taiwan \\ Boris A. Malomed \\ Department of Interdisciplinary Studies, Faculty of Engineering, Tel Aviv University, Tel Aviv 69978, Israel
}

Received June 14, 2005

\begin{abstract}
Quantum photon-number fluctuation and correlation of bound soliton pairs in mode-locked fiber lasers are studied on the basis of the complex Ginzburg-Landau equation model. We find that, depending on their phase difference, the total photon-number noise of the bound soliton pair can be larger or smaller than that of a single soliton, and the two solitons in the soliton pair have a corresponding positive or negative photonnumber correlation. It is predicted for the first time to our knowledge that out-of-phase soliton pairs can exhibit less noise as a result of negative correlation. (C) 2005 Optical Society of America

OCIS codes: $270.5530,140.3510,140.4050$.
\end{abstract}

Quantum solitons have attracted a great deal of research interest in the contexts of nonlinear quantum optics, condensed-matter physics, and quantum information science because of their remarkable nonclassical properties. In particular, quantum solitons in optical fibers largely resemble their classical counterparts but with additional quantum fluctuations around the mean fields. It has been possible to achieve squeezing through quantum solitons in optical fibers, ${ }^{1-4}$ and they may also serve as a new platform for quantum information applications. ${ }^{5-7}$

Quantum solitons are macroscopic optical wave packets that offer a test bed for quantum optics and quantum field theories. For the quantum nonlinear Schrödinger equation (NLSE), exact soliton states can be constructed as combinations of eigenstates of the Hamiltonian of the one-dimensional Bose gas with $\delta$-like (contact) interaction through the Bethe ansatz method. ${ }^{4}$ In the large photon-number limit, which corresponds to the usual optical solitons generated by lasers, the many-photon wave function of the quantum soliton is well approximated by a single-photon wave function (the Hartree approximation). ${ }^{3}$ Linearization around such a soliton $^{8,9}$ successfully explains experimental observations of quantum fluctuations for temporal fiber solitons, provided that optical loss and higher-order effects are negligible.

It is well known that the force between adjacent solitons in the NLSE model is attractive or repulsive, depending on the phase difference between them. ${ }^{15}$ Stationary bound soliton states in this conservative model do not exist. Formation of effectively stable double-, triple-, and multiple-soliton bound states was predicted in models based on the complex Ginzburg-Landau equation ${ }^{16-18}$ (CGLE) and ob- served experimentally in various passively modelocked fiber lasers. ${ }^{19-21}$ The separation between the solitons in these bound states is quantized, taking a set of discrete values. The amplitude noise in triplet bound states generated by a stretched-pulse ytterbium-doped double-clad fiber laser was observed to be reduced compared with the single soliton pulse. ${ }^{22}$ It is an issue of straightforward interest to study the noise of these bound solitons and to understand why the mode-locked fiber lasers operate more stably in the bound-state regime.

The passively mode-locked fiber lasers are quite accurately described by the cubic-quintic CGLE. In a normalized form, the equation is

$$
\begin{aligned}
i U_{z}+(D / 2) U_{t t}+|U|^{2} U= & i \delta U+i \epsilon|U|^{2} U+i \beta U_{t t} \\
& +i \mu|U|^{4} U-\nu|U|^{4} U,
\end{aligned}
$$

where $U$ is the local amplitude of the electromagnetic wave, $z$ is the propagation distance, $t$ is the retarded time, and $D=+1$ and $D=-1$ correspond, respectively, to anomalous and normal dispersion. Besides the group-velocity dispersion and the Kerr effect, which are accounted for by conservative terms on the lefthand side of Eq. (1), the model also includes the quintic correction to the Kerr nonlinearity, through the coefficient $\nu$, and nonconservative terms. The coefficients $\delta, \epsilon, \mu$, and $\beta$ account for the linear, cubic, and quintic loss or gain, and spectral filtering.

In the CGLE model, with suitable parameters, degenerate bound-state soliton pairs are known to exist through the balance between the gain and the loss, in the form ${ }^{16,17} U(z, t)=U_{0}(z, t+\rho) e^{-i \theta / 2}+U_{0}(z, t-\rho) e^{i \theta / 2}$, where $U_{0}$ is a single-soliton solution and $\rho$ and $\theta$ are the separation and the phase difference between the solitons. In this Letter we focus on the consideration 
of three fundamentally different cases, corresponding to the bound states with the same separation and amplitude, and $\theta=0, \pi / 2, \pi$ (the in-phase, orthogonal, and out-of-phase pair, respectively).

We compute the quantum fluctuations of these soliton pairs by dint of a numerically implemented backpropagation method, ${ }^{23}$ which may be summarized as follows. First, we replace the classical function $U(z, t)$ in Eq. (1) with the quantum-field operator variable, $\hat{U}(z, t)$, which satisfies the equal-coordinate Bosonic communication relations. Next, the equation is linearized around the classical solution through the substitution of $\hat{U}(z, t)=U_{0}(z, t)+\hat{u}(z, t)$, assuming large photon numbers in the solitons. Then a zeromean additional noise operator, $\hat{n}(z, t)$, is introduced to make the quantum perturbation fields in the linearized equation satisfy the Bosonic communication relations (see Ref. 24 for more detail). By imposing suitable correlation functions for the noise operator, the minimum quantum noise in the considered dissipative model is introduced. Therefore the results reported here represent a lower limit required by the fundamental principles of quantum mechanics.

Figure 1 shows the photon-number correlation parameter for the two solitons in the bound soliton pair, which is defined as

$$
C_{12}=\left\langle: \Delta \hat{N}_{1} \Delta \hat{N}_{2}:\right\rangle / \sqrt{\left\langle\Delta \hat{N}_{1}^{2}\right\rangle\left\langle\Delta \hat{N}_{2}^{2}\right\rangle} .
$$

Here, the colons stand for the normal ordering of the operators and $\Delta \hat{N}_{1,2}$ are perturbations of the photonnumber operators for the two solitons, which are numbered $(1,2)$ according to their position in the time domain. Initially, the two solitons are assumed to be uncorrelated, with fluctuations around each soliton obeying the coherent-state statistics. For the inphase pair, the photon-number correlation between the solitons gradually increases to positive values and eventually saturates around $C_{12}=0.36$. But for
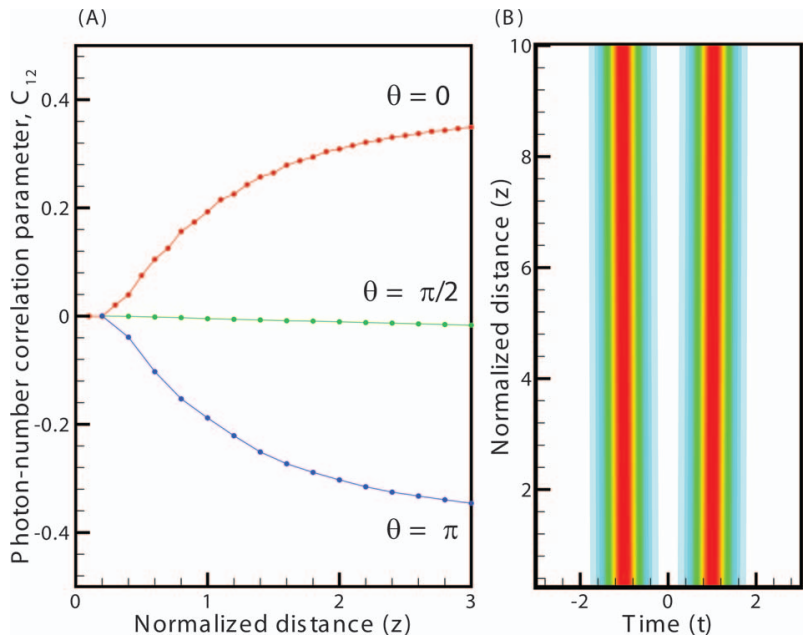

Fig. 1. (A) Photon-number correlation parameter, $C_{12}$, for bound-state soliton pairs with different relative phases. (B) Contour plot of the classical solution for the bound state. All the results are presented for $D=1, \delta=-0.01, \epsilon=1.8, \beta$ $=0.5, \mu=-0.05$, and $\nu=0$ in the CGLE, Eq. (1).
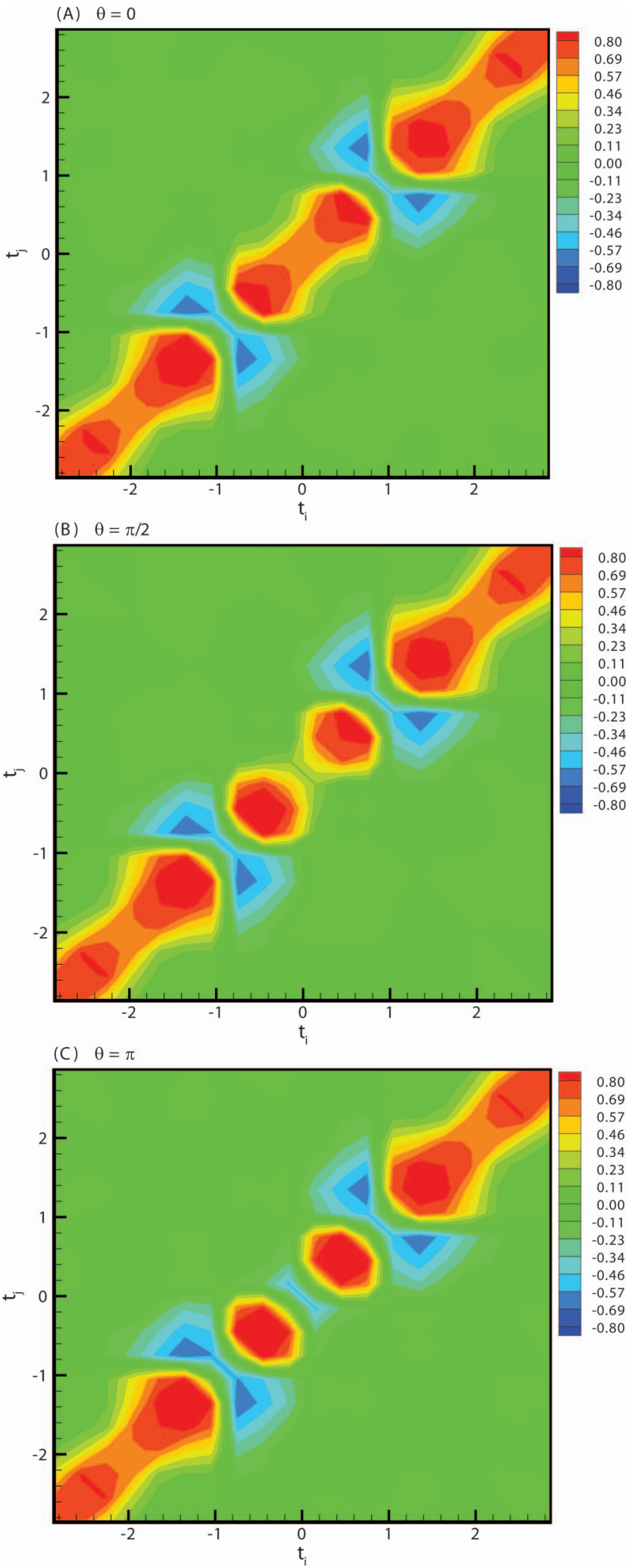

Fig. 2. Time-domain photon-number correlation patterns, $\eta_{i j}$, for bound soliton pairs with different relative phases, after the normalized propagation distance $z=0.4$. (A) $\theta=0$, (B) $\theta=\pi / 2$, and (C) $\theta=\pi$. Time-division length $\Delta t=0.3$.

the out-of-phase pair, $C_{12}$ gradually decreases to negative values and then saturates, too. In between, the correlation parameter for the case of $\theta=\pi / 2$ remains close to zero as long as the computation is run. 
For the former two cases, the saturation of the photon-number correlation parameter is due to the nonconservative effects in the CGLE model.

To further demonstrate the behavior difference of the photon-number correlation for soliton pairs with different relative phases, in Fig. 2 we display the time-domain photon-number correlation patterns for them. The plotted correlation coefficients, $\eta_{i j}$, are defined through the normally ordered covariance,

$$
\eta_{i j} \equiv\left\langle: \Delta \hat{n}_{i} \Delta \hat{n}_{j}:\right\rangle / \sqrt{\Delta \hat{n}_{i}^{2} \Delta \hat{n}_{j}^{2}},
$$

where $\Delta \hat{n}_{j}$ is the photon-number fluctuation in the $j$ th time slot $\Delta t_{j}$,

$$
\Delta \hat{n}_{j}=\int_{\Delta t_{j}} \mathrm{~d} t\left[U_{0}(z, t) \hat{u}^{\dagger}(z, t)+U_{0}^{*}(z, t) \hat{u}(z, t)\right] .
$$

Here the integral is taken over the given time slot, with the same time-division length $\Delta t$. Clearly, in Fig. 2(A) one can see that there is a strong positivecorrelation band connecting the quantum correlation patterns of the bound solitons when they are in phase, $\theta=0$. In Fig. 2(C) there exists a negativecorrelation pattern between two solitons for the outof-phase case, $\theta=\pi$. Moreover, for the case of $\theta=\pi / 2$, in Fig. 2(B), the correlation patterns of bound solitons are almost isolated. In classical physics, inphase and out-of-phase fields will lead, respectively, to constructive and destructive interference. Here we observe a similar effect for the quantum noise. What is more important, in Fig. 3 we compute the total photon number noise of the bound soliton pair and compare it with the case of a single soliton (these results are amenable to straightforward experimental verification). As one may expect, the photon-number noise of the in-phase pair is larger than that for the single soliton, which may be explained as fluctuation enhancement due to constructive interference. On the other hand, the noise is reduced for the out-ofphase pair as the result of destructive interference. The orthogonal soliton pair with $\theta=\pi / 2$ may be

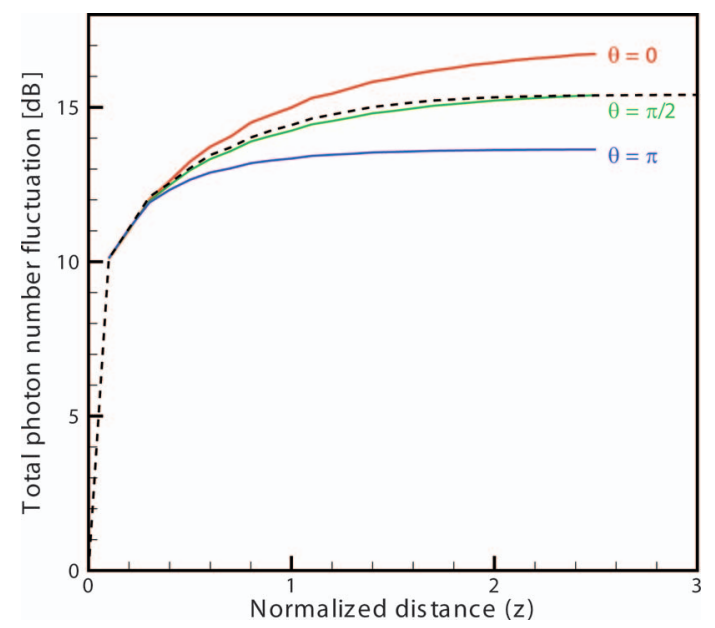

Fig. 3. Comparison of the total photon-number fluctuations in the bound soliton pairs with different relative phases (three solid curves), and around the single soliton (dashed curve). viewed, in the first approximation, as two independent single solitons, which explains why it features almost the same noise level as the single soliton.

In conclusion, we have presented theoretical results for the photon-number correlation and total photon-number noise for bound-state soliton pairs in the model of the complex cubic-quintic GinzburgLandau equation. Cases of in-phase, orthogonal, and out-of-phase soliton pairs have been considered in detail. We conclude that the interference of the quantum fluctuations in the soliton pair is constructive or destructive depending on the classical relative phase of the solitons. An important consequence of the results is that the operation regime of the fiber laser should be more stable when it is based on the out-ofphase soliton pairs.

This work was partly supported by the National Science Council of Taiwan, Republic of China, under projects NSC-94-2215-E-009-019 and NSC-94-2752E-009-009-PAE.

\section{References}

1. S. J. Carter, P. D. Drummond, M. D. Reid, and R. M. Shelby, Phys. Rev. Lett. 58, 1841 (1987).

2. P. D. Drummond and S. J. Carter, J. Opt. Soc. Am. B 4, 1565 (1987).

3. Y. Lai and H. A. Haus, Phys. Rev. A 40, 844 (1989).

4. Y. Lai and H. A. Haus, Phys. Rev. A 40, 854 (1989).

5. Ch. Silberhorn, P. K. Lam, O. Weiss, F. König, N. Korolkova, and G. Leuchs, Phys. Rev. Lett. 86, 4267 (2001).

6. Ch. Silberhorn, N. Korolkova, and G. Leuchs, Phys. Rev. Lett. 88, 167902 (2002).

7. F. König, M. A. Zielonka, and A. Sizmann, Phys. Rev. A 66, 013812 (2002).

8. H. A. Haus and Y. Lai, J. Opt. Soc. Am. B 7, 386 (1990).

9. Y. Lai, J. Opt. Soc. Am. B 10, 475 (1993).

10. M. Rosenbluh and R. M. Shelby, Phys. Rev. Lett. 66, 153 (1991).

11. K. Bergman and H. A. Haus, Opt. Lett. 16, 663 (1991).

12. S. R. Friberg, S. Machida, M. J. Werner, A. Levanon, and T. Mukai, Phys. Rev. Lett. 77, 3775 (1996).

13. D. Krylov, K. Bergman, and Y. Lai, Opt. Lett. 24, 774 (1999).

14. S. Spälter, N. Korolkova, F. König, A. Sizmann, and G. Leuchs, Phys. Rev. Lett. 81, 786 (1998).

15. G. P. Agrawal, Nonlinear Fiber Optics (Academic, 1995).

16. B. A. Malomed, Phys. Rev. A 44, 6954 (1991).

17. N. N. Akhmediev, V. V. Afanasjev, and J. M. SotoCrespo, Phys. Rev. E 53, 1190 (1996).

18. J. M. Soto-Crespo, N. N. Akhmediev, P. Grelu, and F. Belhache, Opt. Lett. 28, 1757 (2003).

19. D. Y. Tang, W. S. Man, H. Y. Tam, and P. D. Drummond, Phys. Rev. A 64, 033814 (2001).

20. N. H. Seong and Dug Y. Kim, Opt. Lett. 27, 1321 (2002)

21. P. Grelu, F. Belhache, F. Gutty, and J. M. Soto-Crespo, J. Opt. Soc. Am. B 20, 863 (2003).

22. B. Ortac, A. Hideur, T. Chartier, M. Brunel, P. Grelu, H. Leblond, and F. Sanchez, IEEE Photon. Technol. Lett. 16, 1274 (2004).

23. Y. Lai and S.-S. Yu, Phys. Rev. A 51, 817 (1995).

24. R.-K. Lee, Y. Lai, and B. A. Malomed, Phys. Rev. A 70, 063817 (2004). 